\title{
Drug reposition as a promising strategy in therapy of cognitive deficits at neurodegenerative disorders
}

\author{
M.A. Tikhonova ${ }^{1,2 *}$, T.G. Amstislavskaya ${ }^{1,2,3}$, Y.J. Ho ${ }^{4}$ \\ ${ }^{1}$ Scientific Research Institute of Physiology and Basic Medicine, Novosibirsk, Russia \\ ${ }^{2}$ Novosibirsk State University, Novosibirsk, Russia \\ ${ }^{3}$ Institute of Cytology and Genetics SB RAS, Novosibirsk, Russia \\ ${ }^{4}$ Chung Shan Medical University, Taichung City, Taiwan, R.O.C. \\ *e-mail: tikhonovama@physiol.ru
}

Key words: cognitive deficits, Parkinson's disease, Alzheimer's disease, neuroinflammation, rats

Motivation and Aim: Drug repositioning (or drug repurposing / drug reprofiling) is a process of redeveloping a compound for application in a different pathology and finding new therapeutic indications for the existing drugs. The premise of repositioning is that the drugs that have previously passed clinical trials will minimize the risk of failure in future late-stage clinical trials due to toxicity and thus lead to faster drug approvals. It has been growing in importance in the last few years and becoming mainstream area in drug research and industry. This strategy appeared to be quite effective approach in psychopharmacology as well. Our project was focused on antimicrobial drug with neuroprotective properties, ceftriaxone (CEF). We evaluated its potential to restore cognitive deficits in animal models of neurodegenerative disorders (Alzheimer's disease (AD), Parkinson's disease (PD)).

Methods and Algorithms: Wistar rats were injected with a selective neurotoxin of dopaminergic neurons MPTP into SNc to establish a PD model. Mice of C57B1/6J strain injected bilaterally i.c.v. with amyloid-beta fragment 25-35 were used as pharmacological AD model while rats of OXYS strain were used as a genetic model of sporadic AD. To evaluate the effects of CEF, the animals were treated with the drug $(100 \mathrm{mg} / \mathrm{kg} / \mathrm{day}$, i.p., 14-36 days) and then underwent behavioral testing for cognitive function. Neuronal activity in vivo was measured with ME-MRI. Brain neuromorphology was evaluated with Nissl staining and immunohistochemical analysis.

Results: The treatment with CEF improved cognitive deficits in MPTP-induced rat PD model, model of sporadic AD in OXYS rats with accelerated senescence, and murine AD model induced by i.c.v. A $\beta$ injections. We showed that MPTP lesioning resulted in decreased neuronal activity and density in the nigrostriatal dopaminergic (DAergic) system and the hippocampal CA1, CA3, and dentate gyrus, while CEF treatment prevented those disturbances. Neuromorphologically, control OXYS rats exhibited a lowered neuronal density in the hippocampal CA1 area whereas CEF restored this parameter. CEF also de-creased amyloid accumulation and neuroinflammation in $\mathrm{A} \beta$ treated mice.

Conclusion: The results suggest CEF as a promising pharmacological tool for the prevention of cognitive decline at neurodegenerative disorders and gave new insights into mechanisms of its neuroprotective effects.

Acknowledgements: Supported partially by grants No. 15-04-05593-a, 15-54-52029 HHC-a from the Russian Foundation for Basic Research (Russia) and No. MOST 104-2923-H-040-001-MY3 from the Ministry of Science and Technology (Taiwan, R.O.C.); by NSU: Academic Strategic Unit "Neuroscience in Translational Medicine". 